\title{
Optical sampling technique applied to high resolution distributed fibre sensors
}

\author{
Stella Foaleng-Mafang ${ }^{1}$, Jean-Charles Beugnot and Luc Thévenaz \\ EPFL Swiss Federal Institute of Technology,Group for Fibre Optics, Institute of Electrical \\ Engineering, STI IEL GR-SCI Station11, CH-1015 Lausanne, Switzerland
}

\begin{abstract}
A technique developed to acquire fast optical signals using low frequency detection and acquisition is presented here. It is based on optical sampling that creates a replica of the fast signal on a much slower time scale by a simple strobe effect. High bandwidth detection and acquisition is totally suppressed leading to a better response and a substantial cost reduction. The performance is illustrated by comparative measurements using a Brillouin high resolution distributed fibre sensor.
\end{abstract}

Keywords: Optical sampling; distributed fibre sensor; optical signal processing, Brillouin scattering .

\section{INTRODUCTION}

During the past decades, a substantial effort has been carried out for developing distributed fibre sensors, working towards longer range and better spatial resolution. In classical time-domain techniques the spatial resolution is given by the temporal width $\tau$ of the pulse activating the measuring process. As a result of the group velocity in an optical fibre a $1 \mathrm{~m}$ spatial resolution corresponds to a $10 \mathrm{~ns}$ pulse width. The detection stage must offer a sufficient bandwidth $B W$ to resolve a point change that will have span over a distance given by the pulse width. A traditional relationship from signal theory fixes the minimum bandwith necessary to resolve the pulse as $B W=1.5 / \tau$, so that a $1 \mathrm{~m}$ spatial resolution requires at least a $150 \mathrm{MHz}$ bandwidth in the detection stage.

Identically the data acquisition requires a minimum sampling rate to resolve all changes in the signal activated by the pulse and returned from the fibre to the detector. The Nyquist-Shannon theorem provides the condition that the sampling rate $f_{s}$ must be at least equal to twice the maximum frequency contained in the signal, thus $f_{s}=2 B W=3 / \tau$. A $1 \mathrm{~m}$ spatial resolution will thus requires a minimum sampling rate $f_{s}=0.3$ Gigasample/s in the acquisition stage.

Recently novel configurations showing sub-metric spatial resolutions have been proposed [1-3] that require pulse duration of $1 \mathrm{~ns}$ and shorter. The detection and acquisition stages must be adapted accordingly, resulting in bandwidth of several GHz and sampling rate of many Gigasample/s. This has of course a very large impact on the cost of the system, since the needed devices enter in a superior category in terms of performance, with a price multiplied by a very substantial factor.

Even worse, the response and the noise of the detection stage also degrade proportionally to the bandwidth, while the sensor response also decays linearly with decreasing pulse widths. These combined effects make the SNR of the global sensor response to depend on the pulse width as $\tau^{3 / 2}$ in identical experimental conditions.

To alleviate this issue we here propose a technique that offers the possibility to detect and sample a signal covering a bandwidth of several $\mathrm{GHz}$ using an arbitrary low bandwidth detection stage and a low sampling rate acquisition card. The potentiality and the relevance of the technique are illustrated on a Brillouin optical time domain analyzing system (BOTDA), by comparing the response given by a classical direct detection and the optical sampling technique. To our knowledge this is the first time that the optical sampling technique is proposed in distributed fibre sensors.

\section{PRINCIPLE OF OPTICAL SAMPLING}

Fig. 1 depicts the principle of the optical sampling technique [4]. The upper part of the figure shows an optical signal which can be for instance a repetitive signal with period $T_{1}$. Before detection this periodical signal passes through a fast

\footnotetext{
${ }^{1}$ Email: stella.foalengmafang@epfl.ch; phone+41 21693 7387; fax +41 216934660
} 


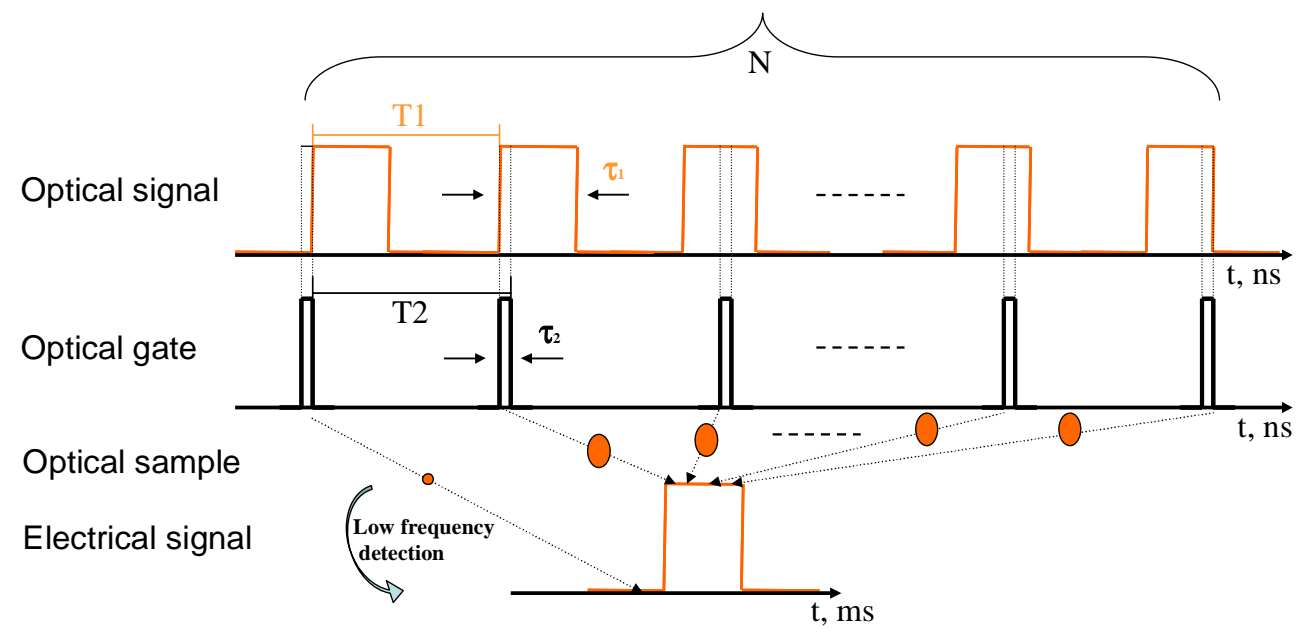

Fig. 1: Principle of optical sampling

optical gate that is opened with a periodicity $T_{2}$, chosen close but different to $T_{1}$. The gate is in off-state most of the time except for brief time intervals $\tau_{2}$. If the width $\tau_{2}$ of the optical gate is much shorter than the typical duration of the optical signal $\tau_{1}$, as shown in Fig. 1, only a brief fraction of the optical signal is sliced out in the time domain. A sample of duration $\tau_{2}$ is so sliced from the optical signal and is transmitted to the detector. Since the sampling periodicity is slightly different to the signal period a sample shifted in time is extracted at each signal period. After a low pass filtering a replica of the original signal is obtained on a multiplied time scale, in full similarity with a stroboscopic effect.

Let $N$ be the number of time resolved points to extract from the signal in the time domain. The sampling period $T_{2}$ can be expressed as follows:

$$
T_{2}=T_{1}+\frac{T_{1}}{N}
$$

This means that after each period $T_{1}$, the optical gate is time-shifted by $T_{1} / N$. If $T_{\text {acq }}$ is the real time necessary to display the signal replica on the scope after sampling and acquire it, then $T_{\text {acq }}=N . T_{1}$ and $N$ true signal periods are required to scan the entire signal.

If the signal must be displayed and acquired only over a time window $T_{e}<T_{1}$ randomly positioned along the entire waveform, $T_{2}$ is expressed in this case as follows:

$$
T_{2}=T_{1}+\frac{T_{e}}{N}
$$

The parameter $T_{1}$ is fixed by the total length $L$ of the sensing fibre and is given by the following relation: $T_{1}>\frac{2 L}{V_{g}}$, where $V_{g}$ is the group velocity of the light in the fibre.

Fully sampling the signal over the analyzing window requires a burst of $N$ sampling pulses and the repetition frequency $f_{B}$ of the burst sequence must satisfy the condition $f_{B} \leq 1 / T_{a c q}$ to avoid any temporal superposition.

The proper setting of the sampling technique requires a special care, suggesting some comments and guidelines:

- The gate window $\tau_{2}$ must be shorter than the activating pulse width $\tau$ to preserve the instrumental spatial resolution.

- The acquisition time $T_{a c q}$ depends on the fibre length $L$ that fixes the signal repetition rate $T_{1}$, as in a classical detection scheme, but also on the number of sampling points $N$ since $T_{\mathrm{ac} q}=\mathrm{N} \cdot T_{1}$.

- The low pass filtering must be low enough to smooth the response from the gating device, but large enough to observe a change at each sample step. These 2 competing conditions result in a strict value for the bandwidth of the detection stage that must be exactly $B W_{\text {det }}=0.35 / T_{1}$.

To give an example how the system must be properly set, the parameters selected for the illustrative measurements shown below were calculated as follow.

$$
L=2.2 \mathrm{Km} \rightarrow T_{1}=T_{e}=25 \mu \mathrm{s} \rightarrow B W_{\mathrm{det}}=14 \mathrm{kHz} \quad \mathrm{N}=2500 \rightarrow T_{2}=25.010 \mu \mathrm{s} ; T_{\text {acq }}=62.5 \mathrm{~ms} ; f_{B}=13 \mathrm{~Hz}
$$




\section{EXPERIMENTAL SETUP AND RESULTS}

Fig. 2 represents the experimental set-up used for demonstrating the potentiality of the optical sampling technique. The black box part represents a classical time-of-flight distributed measurement system, in the present case a low noise Brillouin optical time domain analyzer (BOTDA) described in detail in [5]. Generator-1 supplies the activating pump pulses of width $\tau$. The second part describes the specific sampling configuration where a semiconductor optical amplifier (SOA) is used to create an optical gate by delivering bursts of short electrical pulses (width $\tau_{2}$ ) on its electrodes supplied by Generator-2. The gate window $\tau_{2}$ was set to $8 \mathrm{~ns}$ which is the shortest pulse width supplied by Generator- 2 . Time jitter was minimized by using a highly stable $10 \mathrm{MHz}$ master clock to drive both generators.

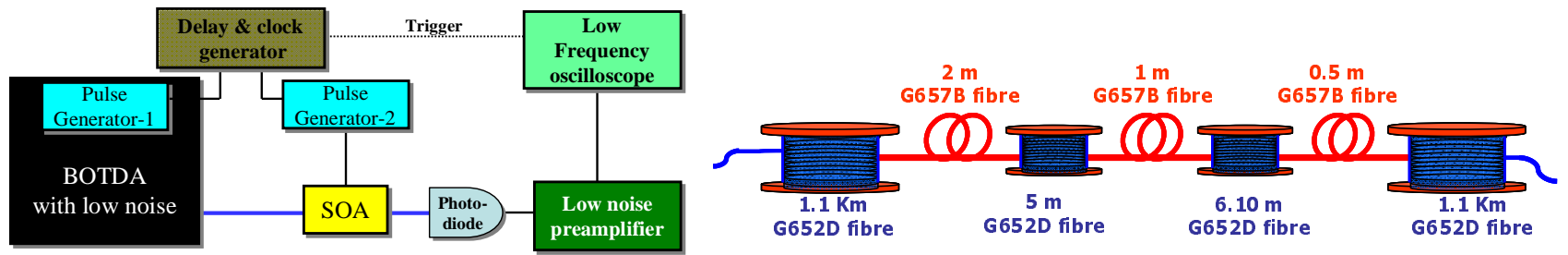

Fig. 2: Complete set-up for optical sampling distributed Fig. 3: Fibre arrangement used for the comparative tests. At a Brillouin sensing. wavelength of $1557 \mathrm{~nm}$ the Brillouin resonance of the fibres is $v_{B}=10.693 \mathrm{GHz}$ for the G652D fibre and $v_{B}=10.520 \mathrm{GHz}$ for the G657B fibre.

A low frequency detection stage was used to allow a high gain and low noise detection for the optical signal. The gain of the preamplifier was set, so that the bandwidth of the detection stage exactly matches the bandwidth required for the acquisition. The sampled and filtered signal is then visualized on a low frequency oscilloscope triggered by the master clock.

In the BOTDA system the pump pulse width $\tau$ was set to $20 \mathrm{~ns}$ ( $2 \mathrm{~m}$ spatial resolution); therefore the $8 \mathrm{~ns}$ gate width $\tau_{2}$ does not limit the spatial resolution. A special test fibre arrangement made of $2 \mathrm{~m}, 1 \mathrm{~m}$ and $0.5 \mathrm{~m}$ sections of G657B fibres placed in the middle of a $2.2 \mathrm{~km} \mathrm{G652D}$ fibre, as depicted in Fig. 3, was analyzed. The Brillouin frequency of the short sections is shifted by $173 \mathrm{MHz}$ compared to the rest of the fiber.

The time traces shown in Fig. 4 represent the local Brillouin gain measured at the resonance frequency in the G652D fibre. Fig. 4a represents a measurement performed by a classical detection scheme using a $125 \mathrm{MHz}$ bandwidth detector and Fig. $4 \mathrm{~b}$ the same Brillouin gain signal obtained by optical sampling using a low frequency detector $(100 \mathrm{kHz})$ and a low performance digital oscilloscope. The fluctuations observed in the two traces must not be assimilated to noise; they are due to the fact that SBS is inherently dependent on the polarization. The aspect of the two traces is very similar and the noise looks much better controlled in the case of the optical sampling. The time scale is expanded over more than 3 decades and the original time value can be easily retrieved from the relations given in section 2 by simply dividing the acquisition time scale by $N$.

To check the adequacy of the optical sampling technique with a complete Brillouin analysis and the possibility to measure an arbitrary time window, measurements have been realized using the same test fibre by scanning the signal
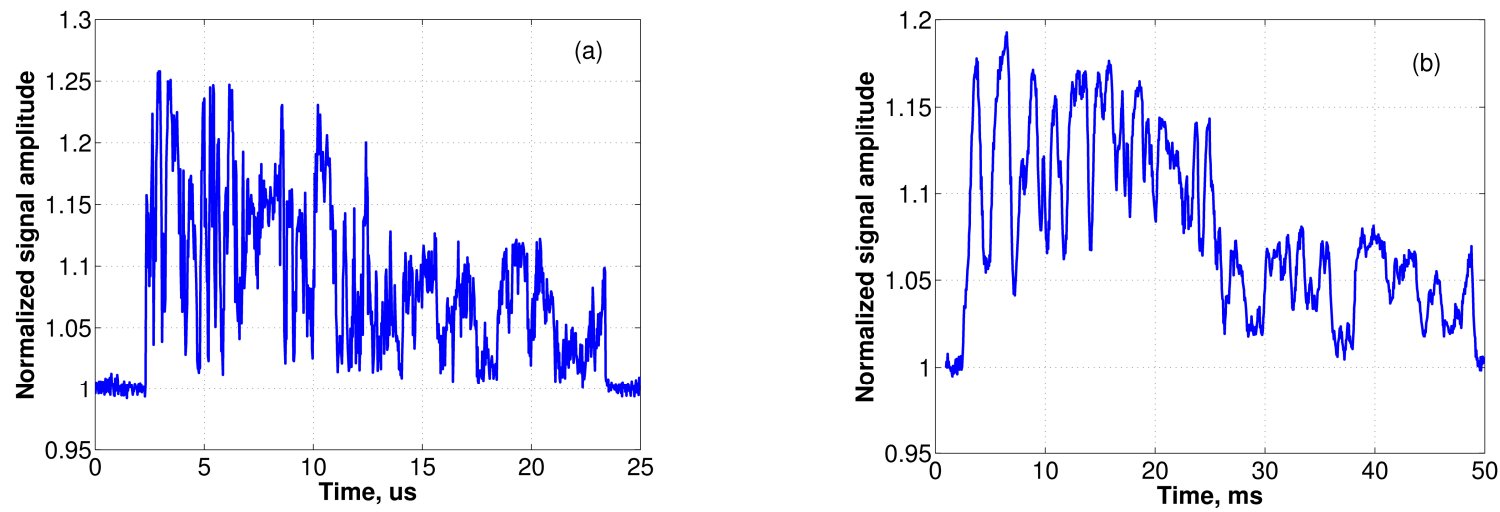

Fig. 4: (a) Brillouin gain as function of the position in the fibre measured using direct detection and a high frequency detector; (b) the corresponding optical signal measured using the optical sampling technique and a low frequency detector. 
frequency around the Brillouin Stokes frequency of the two types of fibre (G657B and G652D) and by restricting the acquisition on a time window centered on the $2 \mathrm{~m}, 1 \mathrm{~m}$ and $0.5 \mathrm{~m}$ fibre segments.

Fig. 5 represents the 3D Brillouin gain distribution as a function of frequency and distance along the fibre. The measurement performed with a $2 \mathrm{~m}$ spatial resolution clearly shows the presence of the Brillouin frequency shifted segments. The $2 \mathrm{~m}$ section is fully resolved, while the $1 \mathrm{~m}$ and $0.5 \mathrm{~m}$ sections are only partially resolved but still clearly detected.

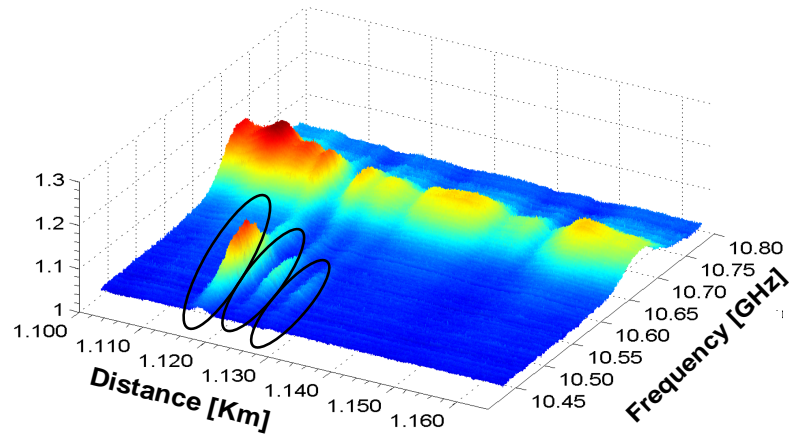

Fig. 5: 3D plot of the distribution of the Brillouin gain as a function of distance and frequency of the central region of the fibre arrangement shown in Fig. 3. The short segments made of distinct fibres are clearly visible. The acquisition is entirely realized using the optical sampling technique with a 64 times averaging.

\section{CONCLUSION}

The possibility to use the optical sampling technique for distributed fibre measurements at high spatial resolution has been demonstrated. This technique can offer an attractive solution to circumvent the problem of fast electronics that is not widely available, expensive and limited in performance in terms of sensitivity and noise. The only fast electronic device is the pulse generator that must be capable to generate pulses at the sampling resolution.

The optical sampling technique offers the advantage of optimizing the bandwidth and noise performance of the system, and consequently to maximize the available gain in a given configuration.

A rapid analysis of the technique shows that the effective electrical signal at the output of the detection stage is of similar amplitude for the direct and the sampling techniques: the amount of light on the detector is reduced by a factor equal to the duty cycle of the optical gating, but the gain on the pre-amplification stage can be raised in the same proportion as a result of the bandwidth reduction. As far as the noise is concerned, the limited bandwidth leads to a massive reduction of the noise level.

A critical point is the acquisition time that is clearly extended as a result of the time scale expansion. A simple inspection of the situation shows that the noise reduction results in a reduced number of averaging that exactly compensates the extra acquisition time when compared to the direct detection technique. It can thus be concluded that the optical sampling technique does not cause any impairment in performance if it is properly set up and brings the decisive benefit to improve the potential resolution of a distributed sensor without requiring an advanced instrumentation.

Certainly optical sampling can offer an attractive solution for the next generation of high spatial resolution optical fibre sensors.

\section{ACKNOWLEDGMENTS}

The authors would like to acknowledge the support from Omnisens SA and the tight and supportive collaboration with ETHZ-IGT.

\section{REFERENCES}

1. X. Bao, A. Brown, M. DeMerchant, and J. Smith, "Characterization of the Brillouin-loss spectrum of single-mode fibers by use of very short (10-ns) pulses", Opt. Lett., 24, 510-512, 1999.

2. Anthony W. Brown and Bruce G. Colpitts, "Dark-Pulse Brillouin optical Time-Domain Sensor with 20-mm Spatial Resolution" JLT, 25, 381-386, 2007.

3. L. Thévenaz, S. Foaleng Mafang, "Distributed fiber sensing using Brillouin echoes", 19th International Conference on Optical Fibre Sensors, (SPIE, Perth, WA, Australia), pp. 70043N-70044, 2008.

4. M. Niklès, L. Thévenaz, P. A. Robert, and J. P. von der Weid, "Optical Sampling using Wideband ElectroOptic Modulators", Opt. Eng, 34, 2078, 1995.

5. S. Diaz, S. Foaleng Mafang, M. Lopez-Amo, and L. Thevenaz, "A High-Performance Optical Time-Domain Brillouin Distributed Fiber Sensor," IEEE Sensors Journal, 8, 1268-1272 (2008). 